\title{
KNEE PAIN AND POSTURAL STABILITY IN WOMEN WITH GONARTHROSIS BEFORE AND SIX MONTHS AFTER UNILATERAL TOTAL KNEE REPLACEMENT
}

\author{
M. Rätsepsoo ${ }^{1,2}$, H. Gapeyeva ${ }^{1,2}$, D. Vahtrik ${ }^{1,2}$, H. Aibast ${ }^{1,2}$, \\ J. Ereline ${ }^{1,2}$, T. Haviko ${ }^{3}$, A. Märtson ${ }^{3}$, M. Pääsuke ${ }^{1,2}$ \\ ${ }^{1}$ Institute of Exercise Biology and Physiotherapy, \\ University of Tartu, Tartu, Estonia \\ ${ }^{2}$ Centre of Behavioral and Health Science, \\ University of Tartu, Tartu, Estonia \\ ${ }^{3}$ Department of Traumatology and Orthopaedics, \\ University of Tartu, Tartu, Estonia
}

\begin{abstract}
The aim of this study was to investigate knee pain and postural stability in women with knee osteoarthritis (OA), who used postoperative home exercise program (HEP) with balance exercises. 14 women with knee OA in stage III-IV (aged 48-70 years) participated in this study before and 6 months after unilateral total knee arthroplasty (TKA). All patients performed HEP during 6 months after unilateral TKA. Data of patients was compared with healthy age-matched women (controls, $\mathrm{n}=10$ ). Postural stability characteristics (centre of pressure (COP) displacement in the anterior-posterior (AP) and mediolateral (ML) direction, COP sway equivalent radius and area) during $30 \mathrm{~s}$ bipedal standing (eyes open) were recorded on two dynamographic force plates. Pain in knee joint was estimated with visual-analogue scale. COP displacement in AP direction of the operated and non-operated leg was greater $(p<0.05)$ in women with knee OA before and 6 months after TKA compared to the controls. Knee joint pain in the operated leg reduced $70 \%$ after TKA. TKA together with HEP has an important role in preserving postural stability and reducing knee joint pain in women with III-IV stage gonarthrosis.
\end{abstract}

Key words: knee osteoarthritis, static balance, postural sway, rehabilitation. 


\section{INTRODUCTION}

Osteoarthritis (OA) is the most common joint disease worldwide [18] that appears to originate in the cartilage and affects the underlying bone, soft tissues, and synovial fluid and is mostly affecting the hand and large weight-bearing joints such as the knee [5]. It is particularly disabling when the knees are affected, because it limits the ability to walk, to rise from a chair, and to use stairs [8].

One of the main indications of knee joint OA is pain that is incompatible with normal daily living and that does not respond to conservative treatment [10]. Patients with greater preoperative pain have more postoperative pain [2]. Hassan et al. [11] observed that knee joint pain and quadriceps strength were significant predictors of increased postural sway. Women who reported widespread pain had a $60 \%$ greater risk of falling compared to women with no or mild pain [17].

Balance is an integral component of many activities of daily living [12]. It has been found that compared with healthy subjects, patients with knee joint OA have poor postural stability, which is associated with a decrease in functional performance [14]. Pain and muscle strength may particularly influence postural sway [11]. Impairments in knee joint proprioception have been mentioned by multiple authors $[11,14,21]$. These deficits, in combination with the ageing process, may culminate in greater impairments of balance in this patient population, compared with their age-matched and healthy counterparts [12].

Total knee arthroplasty (TKA) is the most common treatment for stage III-IV gonarthrosis [16]. Patients with TKA also have impaired balance and movement control [19]. Osteoarthritis pathology and TKA appear to alter the proprioceptive function of the knee [6] as well as soft-tissue release during total knee replacement [1]. Changes in the proprioceptive function of the knee joint may contribute to altered balance control during standing and walking [6].

Study of Piva et al. [19] was to determine the feasibility of balance exercise program in patients with TKA. They found that improvement was higher for gait speed, single-leg stance time, and knee joint stiffness. Research of home exercise programs with prevalence of balance exercises to improve postural stability in women with gonarthrosis has not been enough elucidated in literature. The aim of the present study was to (1) investigate postural stability and knee 
joint pain in women with gonarthrosis who used postoperative home exercise program (HEP) with balance exercises before and 6 months after unilateral TKA and to (2) analyze the relationship between these parameters and (3) compare the results with healthy age-matched controls.

\section{MATERIALS AND METHODS}

\section{Subjects}

14 women (mean age 60.2, $\mathrm{SE} \pm 2.0$ years; mean body mass 88.6, $\mathrm{SE} \pm 3.6 \mathrm{~kg}$; mean height 160.0, $\mathrm{SE} \pm 1.5 \mathrm{~cm}$; mean $\mathrm{BMI} 34.7, \mathrm{SE} \pm 1.4$ ) with knee OA in stage III-IV by Kellgren and Lawrence [16] and 10 healthy women (controls, mean age $59.5, \mathrm{SE} \pm 2.1$; mean body mass $66.9, \mathrm{SE} \pm 3.8 \mathrm{~kg}$; mean height 160.0, $\mathrm{SE} \pm 2.0 \mathrm{~cm}$; mean BMI 26.7, $\mathrm{SE} \pm 1.6)$ participated in this study. The patients were operated at Tartu University Hospital. A condylar prosthesis GEMINI (W. Link Gmbh and Co., Germany) was used and seven out of 14 subjects underwent right knee TKA. Average duration of knee joint pain was 6.5 years. Each patient received a home exercise program (HEP) which contained knee range of motion exercises, strengthening and stretching exercises [4], movement control exercises, balance exercises and coordination exercises [13]. Additionally walking, bicycling and swimming were recommended. Each subject filled out an exercise diary.

Control group included 10 healthy age-matched women, who were not diagnosed with gonarthrosis and were moderately physically active.

Criteria for excluding subjects from both groups were: other musculoskeletal diseases, neurological diseases and diseases effecting balance and coordination. All of the subjects signed a written consent to participate in the study voluntarily. Ethics Committee of the University of Tartu approved the study. The research was conducted at the Laboratory of Kinesiology and Biomechanics of University of Tartu from September 2009 to March 2011. Patients participated in the study twice ( 1 day before surgery and 6 months after surgery), controls once. 


\section{Measures}

For postural stability assessment subject was asked to stand on two dynamographic force plates Kistler 9286A (Szwitserland) during 30 seconds with right and left leg on different platform (distance between feet was $20 \mathrm{~cm}$ ) and eyes open. Following body sway characteristics were recorded by movement analysis system Elite Clinic and SWAY ${ }^{\circledR}$ software (BTS S.p.A.): (1) COP sway displacement in the anteriorposterior (AP) (mm) and mediolateral (ML) (mm) direction; (2) COP equivalent radius $(\mathrm{mm})$ and COP equivalent area $\left(\mathrm{mm}^{2}\right)$. All of the characteristics were normalized to the subject's height $(\mathrm{m})$ and foot length $(\mathrm{mm})$ [3].

Knee joint pain was assessed by visual-analogue scale (VAS) in both legs, where 0 - pain is absent and 10 - maximal pain.

\section{Statistical analysis}

Data is presented by means and standard errors (SE). Student paired $t$ test of MS Excel software was used to analyze the differences between pre- and postoperative results and unpaired $t$-test for differences between patients and controls and patients' operated and non-operated leg. Relationship between knee joint pain and postural stability was found by Spearman's rank correlation. $\mathrm{P}$ values $<0.05$ were regarded as statistically significant.

\section{RESULTS}

\section{Knee joint pain}

Knee joint pain (Figure 1) in the operated leg of women with gonarthrosis was significantly higher compared to the non-operated leg as well to controls before TKA $(p<0.001)$ and after TKA $(p<0.05)$. Pain in the operated leg was significantly lower after TKA $(\mathrm{p}<0.001)$ compared to the preoperative state. 


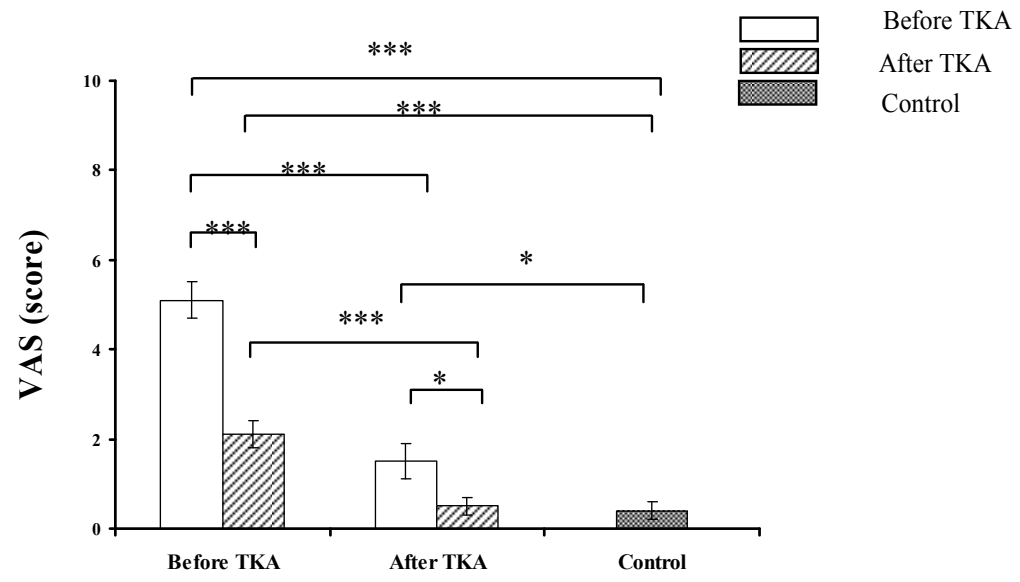

Figure 1. Knee pain in women with gonarthrosis before and 6 months after TKA compared to healthy controls (average of both legs) $($ mean \pm SE). $* * * p<0.001 ; * \mathrm{p}<0.05$.

\section{COP displacement}

COP displacement characteristics in AP direction (COP AP) (Figure $2 \mathrm{~A}$ ) on the operated leg in women with gonarthrosis were greater compared to the non-operated leg before TKA, but the difference was not statistically important. The results in the operated leg were significantly greater compared to controls $(p<0.01)$. A tendency of improvement (decrease) in COP AP results in patients' operated leg compared to preoperative status was noted 6 months after TKA. This characteristic of the operated leg after TKA was significantly greater compared to controls $(\mathrm{p}<0.01)$. 

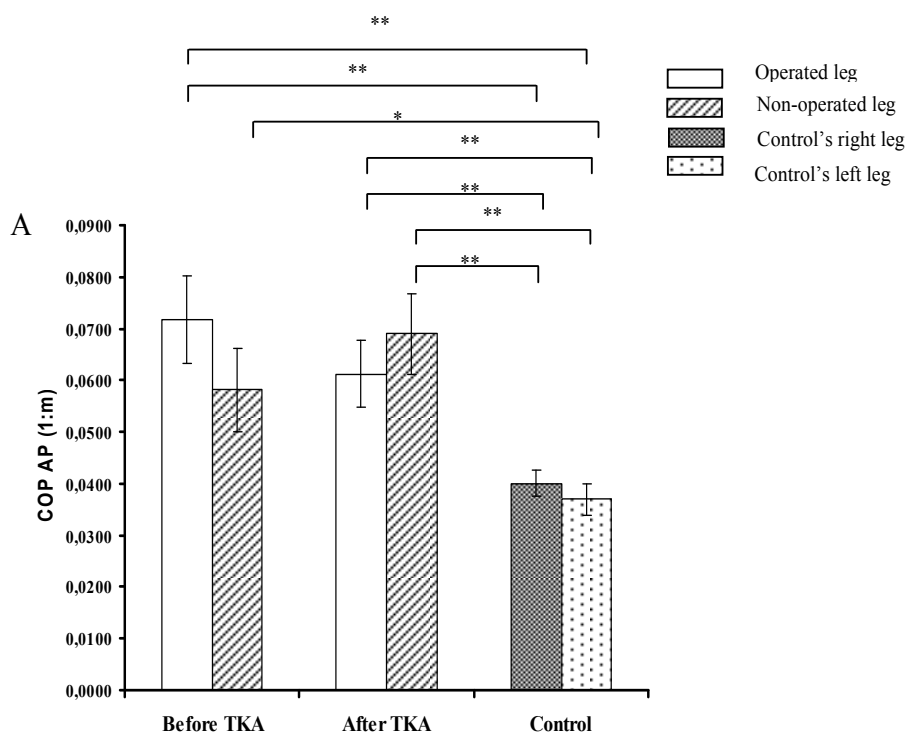

B

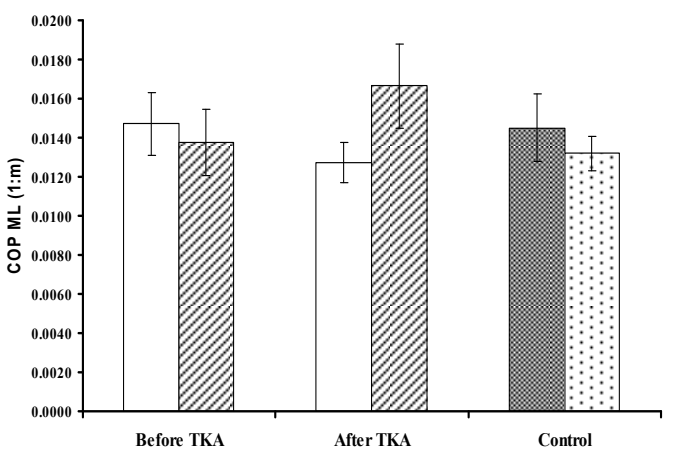

Figure 2. COP sway displacement in anterior-posterior (AP) (A) and mediolateral (ML) direction (B) in women with gonarthrosis before and 6 months after TKA compared to healthy controls (mean \pm SE). Data normalized to subjects height $(\mathrm{m})$ and foot length $(\mathrm{mm})$. ** $\mathrm{p}>0.01$; $* \mathrm{p}>0.05$

COP displacement in AP direction of patients' non-operated leg were significantly greater $(\mathrm{p}<0.05)$ compared to controls preoperatively and postoperatively. 
Increase of COP displacement in ML direction (COP ML) (Figure 2B) of the operated leg in patients group was preoperatively noted compared to the non-operated leg and controls, but the difference was not statistically significant. A tendency of improvement (decrease) in this characteristic of the operated leg was found 6 months after TKA compared to the preoperative results.

\section{COP sway equivalent area and radius}

$\mathrm{COP}$ equivalent area (Figure $3 \mathrm{~A}$ ) and radius (Figure $3 \mathrm{~B}$ ) of the operated leg in women with gonarthrosis was preoperatively greater compared to the non-operated leg and controls, but the difference was not statistically significant.

A

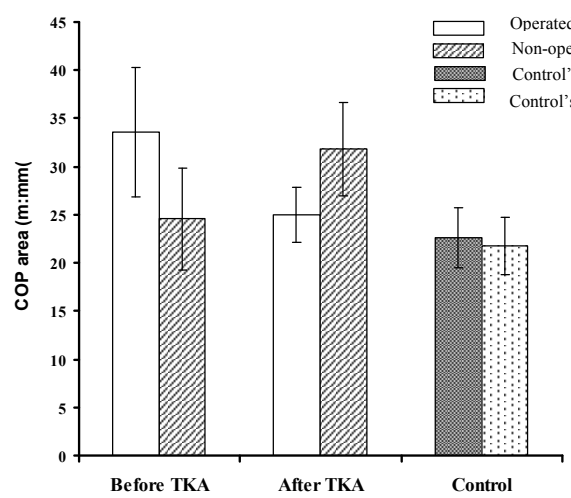

B

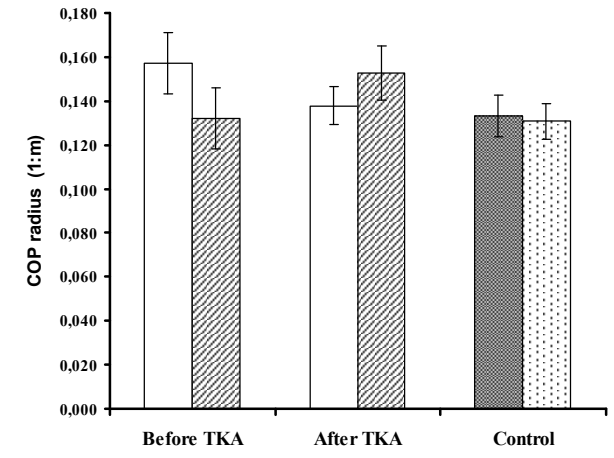

Figure 3. COP sway area (A) and radius (B) in women with gonarthrosis before and 6 months after TKA compared to healthy controls (mean \pm SE). 
A tendency of improvement (decrease) in COP equivalent area and radius characteristics of the operated leg was found 6 months after unilateral TKA compared to the preoperative results. COP sway area and radius of the operated leg were greater compared to the controls, but the difference was not statistically significant.

\section{Correlation}

A medium negative correlation ( $p>0.05$ ) was found between knee pain and postural stability characteristics (Table 1 ).

Table 1. Correlations between knee joint pain and operated leg's postural stability characteristics in women with gonarthrosis before and six months after unilateral total knee arthroplasty.

\begin{tabular}{|l|c|c|}
\hline \multicolumn{1}{|c|}{ Characteristics } & Pain & p \\
\hline COP AP before TKA & 0.17 & NS \\
\hline COP ML before TKA & -0.31 & NS \\
\hline COP AP after TKA & -0.47 & NS \\
\hline COP NL after TKA & -0.46 & NS \\
\hline
\end{tabular}

$\mathrm{COP}$ - centre of pressure, $\mathrm{AP}$ - anterior-posterior, $\mathrm{ML}$ - mediolateral, TKA - total knee arthroplasty, NS - not significant.

\section{DISCUSSION}

Main results of the present study were: (1) postural stability (COP displacement in AP direction) of the operated and non-operated leg in women with knee OA is impaired before and 6 months after TKA compared to the controls, (2) negative moderate correlation ( $p>0.05)$ was noted between knee joint pain and postural stability characters, (3) six months after TKA it is possible to assume that TKA together with home exercise program may conduce to postural stability preservation and knee joint pain reduction in women with gonarthrosis.

OA is the leading chronic condition in the elderly; it affects almost half the population over 65 years old. Joint replacement is the most effective healthcare measure in improving patient quality-of-life outcomes. The need for joint replacements will increase as the population ages [10]. 
Compared with age and sex matched controls, subjects with symptomatic knee OA have quadriceps weakness, reduced knee proprioreception, and increased postural sway (increased lateral sway with eyes open and increased lateral and anterior-posterior sway with eyes closed) [11]. The present study demonstrated that postural stability characteristics in AP direction were significantly greater in women with gonarthrosis compared to healthy controls. No difference was found between operated and non-operated leg data of COP equivalent area and radius in women with gonarthrosis.

Significantly greater sway in the OA group on a firm surface in ML direction has been previously found (eyes open) $[12,13]$. In the present study ML sway decreased $13.4 \%$ after TKA compared to the preoperative result. AP sway of the operated leg decreased $14.8 \%$ after TKA compared to the preoperative result yet was greater compared to the controls (preoperatively $33.9 \%$, postoperatively $22 \%$ ).

In the present study it was found that COP area of the operated leg decreased $25.6 \%$ after TKA but was greater compared to the controls (preoperatively $32.6 \%$, postoperatively $9.5 \%$ ). COP radius of the operated leg decreased $12.4 \%$ after TKA but was greater compared to the controls (preoperatively $15 \%$, postoperatively $3 \%$ ). So it is possible to say that COP area and radius of the patients' operated leg restored to the level close to control group. Tarigan with colleagues [21] found an increase in length of postural sway, postural sway velocity and area of postural sway in patients with knee OA compared with healthy subjects. Swanik et al. [20] found that TKA results in improvements in proprioreception and balance and that postural stability improved 6 months after TKA compared to the preoperative results.

Knee pain is one of the main complications after TKA [7]. In the present study we found that postoperative knee joint pain was reduced by $70 \%$ compared to the preoperative knee joint pain. Brander et al. [2] found that $18.4 \%$ of patients suffer from pain 6 months after TKA. In the present study a significant association between knee pain and postural stability characteristics was not found, but several studies have also suggested an association between knee pain and standing postural instability $[9,11]$. Based on the present study it is possible to speculate that greater knee joint pain in patients with OA who also have flexion contracture may lead to greater postural sway. Also some other characteristics may influence postural stability, such as muscle strength [11]. 
Deficits in components of the balance system and decreased postural control have been identified in OA patients after TKA [20]. Therefore, exercises which aim to improve impaired movement control and balance should be considered in OA patients after TKA [19]. It is possible to conclude that TKA along with HEP may conduce to postural stability preservation and knee joint pain reduction in women with gonarthrosis. The effectiveness of home exercise program has also been found by Jordan et al. [15]. Further studies are necessary to recognize whether postural stability impairments are permanent in women with gonarthrosis or will improve few years after TKA.

\section{ACKNOWLEDGMENTS}

We are grateful to Tartu University Hospital's orthopaedic surgeons for their collaboration and to all the subjects who participated in the present study.

This study was partly supported by the Estonian Ministry of Education and Research project SF0180030s07 and Estonian Science Foundation project 7939.

\section{REFERENCES}

1. Attfield S. F., Wilton T. J., Pratt D. J., Sambatakakis A. (1996) Softtissue balance and recovery of proprioception after total knee replacement. J. Bone Joint Surg. Br. 78: 540-545

2. Brander V. A., Stulberg S. D., Adams A. D., Harden R. N., Bruehl S., Stanos S. P., Houle T. (2003) Predicting total knee replacement pain: a prospective, observational study. Clin. Orthop. Relat. Res. 416: 2736

3. Chiari L., Rocchi L., Cappello A. (2002) Stabilometric parameters are affected by antropometry and foot placement. Clin. Biomech. (Bristol, Avon). 17: 666-677

4. Deyle G. D., Allison S. C., Matekel R. L., Ryder M. G., Stang J. M., Gohdes D. D., Hutton J. P., Henderson N. E., Garber M. B. (2005) Physical therapy treatment effectiveness for osteoarthritis of the knee: a randomized comparison of supervised clinical exercise and manual therapy procedures versus a home exercise program. Phys. Ther. 85: $1301-1317$ 
5. Flugsrud G. B., Nordsletten L., Reinholt F. P., Risberg M. A., Rydevik K., Uhlig T. (2010) Osteoarthritis. Tidsskr. Nor. Laegeforen. 130: 2136-2140

6. Gage W. H., Frank J. S., Prentice S. D., Stevenson P. (2007) Organization of postural responses following a rotational support surface perturbation, after TKA: sagittal plane Rotations. Gait. Posture. 25: $112-120$

7. Garrett B. R., Walters J. (2010) Knee pain, swelling and stiffness after total knee replacement: a survey of South African knee surgeons. SAOJ. 59-66

8. Gür H., Cakin N. (2003) Muscle mass, isokinetic torque, and functional capacity in women with osteoarthritis of the knee. Arch. Phys. Med. Rehabil. 84: 1534-1541

9. Hall M. C., Mockett S. P., Doherty M. (2006) Relative impact of radiographic osteoarthritis and pain on quadriceps strength, proprioception, static postural sway and lower limb function. Ann. Rheum. Dis. 65: $865-870$

10. Hart J. A. (2004) Joint replacement surgery. Med. J. Aus. 180 (5 Suppl): S27-S30

11. Hassan B. S., Mockett S., Doherty M. (2001) Static postural sway, proprioception and maximal voluntary quadriceps contraction in patients with knee osteoarthritis and normal control subjects. Ann. Rheum. Dis. 60: 612-618

12. Hinman R. S., Bennell K. L., Metcalf B. R., Crossley K. M. (2002) Balance impairments in individuals with symptomatic knee osteoarthritis: a comparison with matched controls using clinical tests. Rheumatology (Oxford). 41: 1388-1394

13. Hurley M. V. (2003) Muscle dysfunction and effective rehabilitation of knee osteoarthritis: what we know and what we need to find out. Arthritis Rheum. 49: 444-452

14. Hurley M. V., Scott D. L., Rees J., Newman D. J. (1997) Sensorimotor changes and functional performance in patients with knee osteoarthritis. Ann. Rheum. Dis. 56: 641-648

15. Jordan K. M., Arden N. K., Doherty M., Bannwarth B., Bijlsma J. W., Dieppe P., Gunther K., Hauselmann H., Herrero-Beaumont G., Kaklamanis P., Lohmander S., Leeb B., Lequesne M., Mazieres B., Martin-Mola E., Pavelka K., Pendleton A., Punzi L., Serni U.., Swoboda B., Verbruggen G., Zimmerman- Gorska I., Dougados M. (2003) EULAR recommendations 2003: An evidence based approach to the management of knee osteoarthritis: report of a task force of the standing committee for international clinical studies including therapeutic trials (ESCISIT). Ann. Rheum. Dis. 62: 1145-1155 
16. Kellgren J. H., Lawrence J. S. (1957) Radiological assessment of osteoarthritis. Ann. Rheum. Dis. 16: 494-502

17. Mandeville D., Osternig L. R., Chou L. S. (2008) The effect of total knee replacement surgery on gait stability. Gait Posture. 27: 103-109

18. Michael J. W., Schlüter-Brust K. U., Eysel P. (2010) The epidemiology, etiology, diagnosis, and treatment of osteoarthritis of the knee. Dtsch. Arztebl. Int. 107: 152-162

19. Piva S. R., Gil A. B., Almeida G. J., DiGioia A. M. $3^{\text {rd }}$., Levison T. J., Fitzgerald G. K. (2010) A balance exercise program appears to improve function for patients with total knee arthroplasty: a randomized clinical trial. Phys. Ther. 90: 880-894

20. Swanik C. B., Lephart S. M., Rubash H. E. (2004) Proprioception, kinesthesia, and balance after total knee arthroplasty with cruciateretaining and posterior stabilized prostheses. J. Bone Joint Surg. Am. 86-A: 328-334

21. Tarigan T. J., Kasjmir Y. I., Atmakusuma D., Lydia A., Bashiruddin J., Kusumawijaya K., Prihartono J. (2009) The degree of radiographic abnormalities and postural instability in patients with knee osteoarthritis. Acta Med. Indones. 41: 15-19

\section{Correspondence to:}

Monika Rätsepsoo

Institute of Exercise Biology and Physiotherapy,

University of Tartu

5 Jakobi Street, 51014 Tartu

Estonia

E-mail: monika.ratsepsoo@gmail.com 